\title{
Reply: Misunderstandings in the misconception on the use of pack-years in analysis of smoking
}

J Peto*,1

${ }^{1}$ Department of Non-Communicable Disease Epidemiology, London School of Hygiene and Tropical Medicine, Keppel Street, London WC1E 7HT, UK

Sir,

Lubin and Caporaso's statement (2013) that 'since only packyears estimates the total body burden of the presumed carcinogen, it is the single variable most relevant for characterizing exposure' is both questionable and irrelevant. We want a simple and biologically informative model of the effects of dose and duration of smoking that can be tested and if necessary modified, not a single variable. The most important tests of such models relate to the biological questions they raise, such as whether age per se has any marked effect on cancer incidence owing to this and other human or animal carcinogens, whether smoking affects at least two stages in multi-stage carcinogenesis, and whether a stage is a single genetic event or the abrogation of a pathway involving multiple genes. It is obvious that an incidence rate that equals the square of cigarettes per day multiplied by the fourth power of smoking duration also equals the fourth power of pack-years divided by the square of cigarettes per day. The biological significance of the model is further obscured in the corresponding formula for the relative risk compared with non-smokers, which is the fourth power of pack-years divided by the product of the square of cigarettes per day and the fourth power of age.

\section{REFERENCE}

Lubin JH, Caporaso NE (2013) Misunderstandings in the misconception on the use of pack-years in analysis of smoking. Br J Cancer 108: $1218-1220$. 